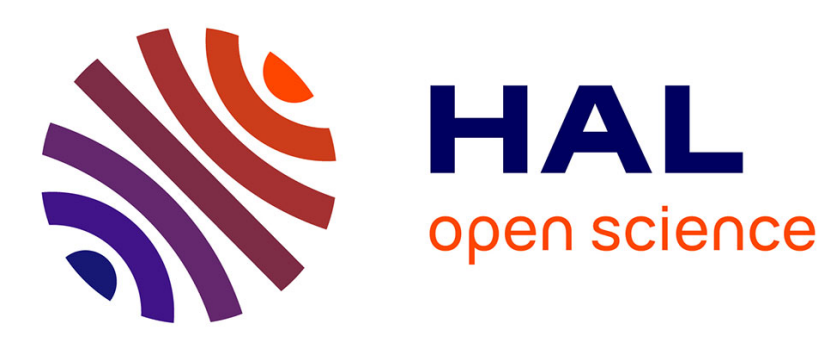

\title{
Periodic billiard orbits in right triangle
}

Serge Troubetzkoy

\section{To cite this version:}

Serge Troubetzkoy. Periodic billiard orbits in right triangle. Annales de l'Institut Fourier, 2005, 55, pp.29-46. hal-00001562

\section{HAL Id: hal-00001562 \\ https://hal.science/hal-00001562}

Submitted on 14 May 2004

HAL is a multi-disciplinary open access archive for the deposit and dissemination of scientific research documents, whether they are published or not. The documents may come from teaching and research institutions in France or abroad, or from public or private research centers.
L'archive ouverte pluridisciplinaire HAL, est destinée au dépôt et à la diffusion de documents scientifiques de niveau recherche, publiés ou non, émanant des établissements d'enseignement et de recherche français ou étrangers, des laboratoires publics ou privés. 


\title{
PERIODIC BILLIARD ORBITS IN RIGHT TRIANGLES
}

\author{
SERGE TROUBETZKOY
}

\begin{abstract}
There is an open set of right triangles such that for each irrational triangle in this set (i) periodic billiards orbits are dense in the phase space, (ii) there is a unique nonsingular perpendicular billiard orbit which is not periodic, and (iii) the perpendicular periodic orbits fill the corresponding invariant surface.
\end{abstract}

\section{INTRODUCTION}

A billiard ball, i.e. a point mass, moves inside a polygon $Q \subset \mathbb{R}^{2}$ with unit speed along a straight line until it reaches the boundary $\partial Q$, then instantaneously changes direction according to the mirror law: "the angle of incidence is equal to the angle of reflection," and continues along the new line. If the trajectory hits a corner of the polygon, in general it does not have a unique continuation and thus by definition it stops there.

Billiards in polygons are easy to describe, but it is difficult to prove deep theorems about them because of a lack of machinery. For example, it is unknown if every polygon contains a periodic billiard orbit. On the other hand, for so called rational polygons, one can apply Teichmüller theory to obtain many deep theorems. It is known that all rational polygons possess many periodic orbits [Ma], and in fact they are dense in the phase space BoGaKrTr. Galperin, Stepin and Vorobets proved many other interesting results about periodic orbits GaStVo. Many of these results can be found in the introductory book by Tabachnikov [Ta], and in several survey articles [Gu1, Gu2, MaTa].

The main result of this article produces the first non rational polygons for which the above mentioned result is true: periodic billiard trajectories are dense in the phase space for an open set of right triangles (Theorem (1). This result is proved by a careful analysis of the symmetries of perpendicular periodic orbits (Theorem 11). Using these symmetries we prove that there is a unique perpendicular escape orbit (Theorem 2) and that the invariant surface which contains the perpendicular direction is completely foliated by perpendicular periodic orbits (Theorem 3). The billiard in a right triangle is well known to be equivalent to the mechanical system of two elastic point particles in an interval (see for example [Ta]), thus our results hold for this system as well. 


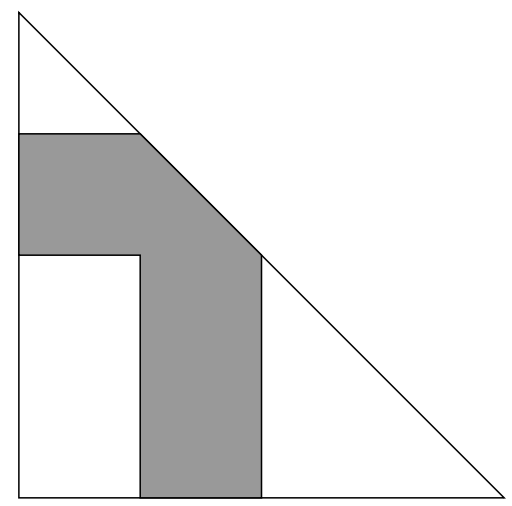

Figure 1. A periodic strip.

\section{Statement of Results}

Cipra, Hanson and Kolan have shown that almost every orbit which is perpendicular to the base of a right triangle is periodic [CiHaKo, Ta]. Here the almost every is with respect to the length measure on the side of the triangle in question. Periodic billiard orbits always come in strips, i.e. for any $x=(q, v)$ whose billiard orbit is periodic where $q \in \partial Q$ and $v$ is any inward pointing direction there is an open interval $I \subset \partial Q$ such that $q \in I$ and for any $q^{\prime} \in I$ the orbit of $x^{\prime}=\left(q^{\prime}, v\right)$ visits the same sequence of sides as $(q, v)$ and thus in particular is periodic (see Figure 1). A maximal width strip will be called a beam. All orbits in a periodic beam have the same period except perhaps the middle orbit which has half the period in the case its period is odd. The results of CiHaKo imply that a set of full measure of the base of the triangle is covered by an at most countable union of intervals such that each of these intervals forms a periodic beam.

They mention "our computational evidence also suggest that the trajectory at the middle of each (perpendicular periodic) beam hits the right-angle vertex of the triangle." This had also been noticed in an earlier article by Ruijgrok [Ru. They also speculate that there is at most one nonsingular, non periodic trajectory. These speculations were the starting point of this research, for this article. I present an elementary proof of these facts for irrational right triangles with smaller angle $\alpha$ satisfying $\frac{\pi}{6}<\alpha<\frac{\pi}{4}$.

Make the convention that one leg of the right triangle is horizontal with the smaller angle $\alpha$ of the right triangle being the angle between this leg and the hypotenuse, and that the word perpendicular (orbit, beam, etc.) refers to perpendicularity to the horizontal leg.

Any billiard trajectory which hits a right-angled vertex (or more generally a vertex with angle $\pi / n$ for some positive integer $n$ ) has a unique continuation. Reflect the triangle in the sides of right angle to obtain a rhombus. The study of the billiard in the triangle reduces 
to that in the rhombus (see next section for details). Throughout the article all beams considered will be with respect to the angle coding in the rhombus defined in the next section.

Theorem 1. For any irrational right triangle whose smaller angle satisfies $\frac{\pi}{6}<\alpha<\frac{\pi}{4}$ consider any perpendicular periodic beam of period $2 p$. Then

(1) the midpoint of the beam hits the right-angle vertex of the triangle (i.e. the mid point of the rhombus),

(2) the beam returns to itself after half its period with the opposite orientation, and

(3) $p$ is even and the first $p+1$ letters of the code of the beam form a palindrome.

The theorem implies that when viewed as an object in the phase space of the billiard flow in the triangle the beam is a Möebius band. This is not in contradiction with the well known construction, of invariant surfaces (see for example Gu1, Ta in the rational case and GuTr in the irrational case) since the directional billiard flow is isomorphic to the geodesic flow on the invariant surface for any direction except for directions which are parallel to a side of the polygon. The direction we are considering is such a direction.

The symmetry of the beam is reminiscent of the symmetry of the beam of periodic orbits around a periodic orbit of odd period mentioned above. Galperin, Stepin and Vorobets showed that any perpendicular orbit whose period is not a multiple of 4 is unstable under perturbation GaStVd. Viewed as an orbit in the rhombus the period is a multiple of 4, however a simple argument shows that the period is not a multiple of 4 when the orbit is viewed as an orbit in a right triangle GaZv.

Call an orbit recurrent if its code $\left\{a_{i}\right\}$ satisfies $a_{j}=a_{0}$ for some $j>0$. Call a nonrecurrent orbit an positive escape orbit if $\lim _{\sup _{i \rightarrow \infty}} a_{i}=\infty$ and negative escape orbit if $\lim _{i \rightarrow \infty} a_{i}=-\infty$. Call a direction simple if there are no generalized diagonals in the invariant surface containing the direction. Here the invariant surface is thought of as arising from the right triangle, so that the vertex at the right angle is considered as a singularity.

\section{Theorem 2.}

(1) Consider an irrational right triangle. In any simple direction then there is at most one non-singular positive escape orbit and at most one non-singular negative escape orbit.

(2) For any irrational right triangle whose smaller angle satisfies $\frac{\pi}{6}<\alpha<\frac{\pi}{4}$, there is at most one non-singular escape orbit in the perpendicular direction. If it exists it is both a positive and negative escape orbit. 

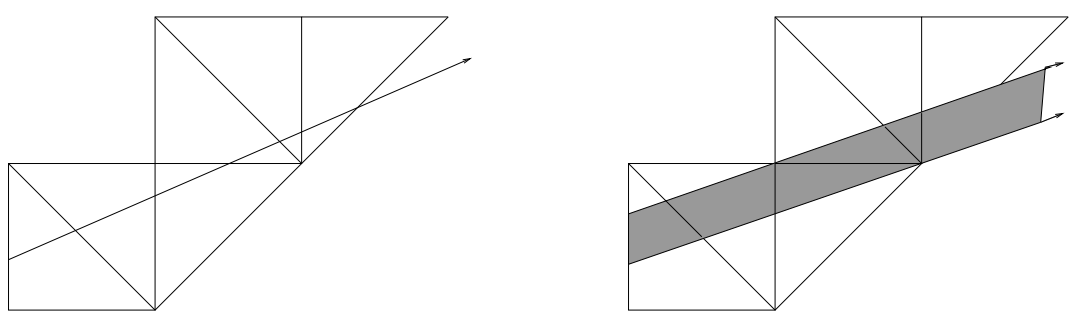

FiguRE 2. Unfolding a billiard trajectory and the associated strip.

If we do not care if the orbit is singular or not, then we can remove the words at most from the statement of the theorem. Theorem 2 is a substantial strengthening of a particular case of a result of $\mathrm{GuTr}$ who show that the set of nonrecurrent orbits has measure zero and of a particular case of a result of [ScTr] who show that this set has lower box counting dimension at most one half.

Boshernitzan has conjectured that given a rational triangle, every nonsingular orbit is periodic in the invariant surface containing a perpendicular direction [B]]. I prove the following analog for irrational right triangles.

Theorem 3. Fix an irrational right triangle whose smaller angle satisfies $\frac{\pi}{6}<\alpha<\frac{\pi}{4}$. Consider the invariant surface of $M$ in the perpendicular direction. Then all the nonsingular orbits on $M$ except the unique escape orbit are periodic.

Combining Theorem 3 with the main theorem of BoGaKrTr yields

Theorem 4. Periodic orbits are dense in the phase space of irrational right triangles whose smaller angle satisfies $\frac{\pi}{6}<\alpha<\frac{\pi}{4}$.

\section{Definitions AND PRoOfs}

There is a nice introductory book on billiards by Tabachnikov Ta and several survey articles Gu1, Gu2, MaTa which can be consulted for details on polygonal billiards in general. For a rational polygon there is a well known construction of invariant surfaces, they are always compact. They same construction leads to noncompact invariant surfaces for irrational polygons. The invariant surfaces for an irrational right triangle can be thought of as having a $\mathbb{Z}$-quasiperiodicity CiHaKo, GuTr, Tr, ScTr, Ta.

The proof is based on the procedure of unfolding of a billiard trajectory. Instead of reflecting the trajectory with respect to a side of a polygon reflect the polygon in this side. Thus the trajectory is straightened to a line with a number of isometric copies of the polygon skewered on it (Figure 2a).

Fix an orbit segment. There is a strip around this trajectory segment such that the same sequence of reflections is made by all trajectories 


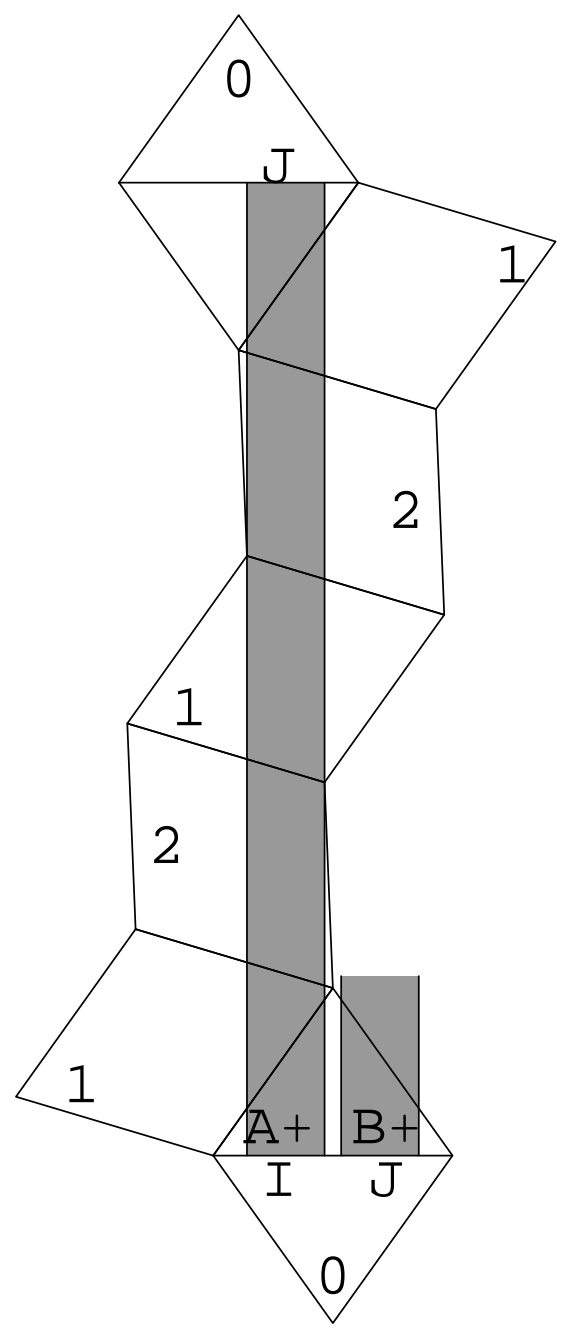

FiguRE 3. A periodic beam in a rhombus with code word 0121210.

in the strip (Figure $2 \mathrm{~b}$ ). The number of reflections is called the length of the strip. Call a maximal width strip a beam. The boundary of a periodic beam consists of one or more trajectory segments which hit a vertex of the polygon. If this vertex is the right-angle one, then the sequence of reflections on both sides of it is essentially the same since the singularity due to such a vertex is removable. This enables us to consider the billiard inside a rhombus which consists of four copies of the right triangle and unfold the rhombus (Figure 3). The central and reflectional symmetries of the rhombus play an essential part in the proofs of the theorems.

Throughout the article unfoldings will be of the billiard in the rhombus and thus billiard orbits through its center will be considered as defined. As we follow the reflections along a straight line trajectory we 


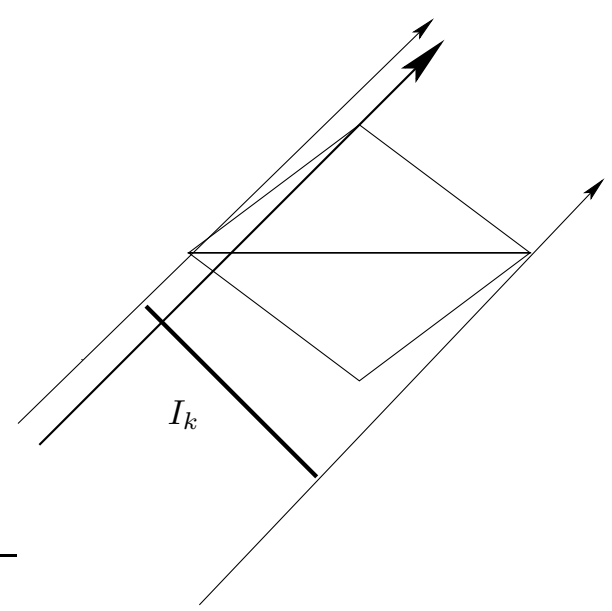

FiguRE 4 . Rhombus $k$ projectivized to the interval $I_{k}$.

see that each flip rotates the rhombus by $2 \alpha$ where $\alpha$ is one of the interior angles of the rhombus. Thus we can label the rhombi with integers according to the total number of (clockwise) rotations by $2 \alpha$. Each orbit is coded by the sequence of labelled rhombi it visits, for example any orbit in the beam $A^{+}$in Figure 3 has code 0121210. It is often convenient to consider labelled rhombus in a projective sense as an interval, namely for rhombus $k$ consider the beam of length 1 with code $k$ and then the associated interval $I_{k}$ is just the perpendicular width of of the beam (see Figure 4 ). The $k$ th-rhombus or the associated interval $I_{k}$ will often be referred to as level $k$. All reference to the length of a beam will pertain to the number of rhombi it crosses which differs from its length in the right triangle.

Let us begin with the following fact.

Lemma 5. Suppose the $A^{+}$and $B^{+}$are parallel beams (not necessarily perpendicular or periodic) with the same codes, then $A^{+}=B^{+}$.

Proof. The proof is by induction. Fix an initial direction. The base case is clear, there is a single beam with code 0 . Furthermore, there is a single beam with code 01 , a single beam with code $0\left({ }^{-} 1\right)$ and these two beams of length two are separated by an orbit which hits a vertex of the rhombus. Assume that every beam with length $n$ is the unique beam with its code. Fix a beam of length $n$ and denote by $j$ the last rhombus it visits. The $j$ th rhombus is attached to the $(j+1)$ st by two parallel sides and to the $(j-1)$ st along the other two parallel sides. There are two points which are common to the $(j-1)$ st, $j$ th and $(j+1)$ st rhombi. During a given pass through the $j$ th rhombus, the beam can hit only one of the two in exiting the $j$ th rhombus (it can hit the other in entering the $j$ rhombus). The orbit which splits a beam is emphasized in Figure 4 . If the beam does not arrive at this point then it does not split and the beam can be continued to a unique beam of 
length $n+1$. If the beam reaches this point then its continuation is split into two sub-beams of length $n+1$ with the resulting sub-beams code differing in the $(n+1)$ st place.

Next we need the following fact.

Lemma 6. The three statements in Theorem 1 are equivalent.

Proof. Perpendicular periodic beams must have even period since they must retrace their orbits between the two perpendicular hits. Fix a perpendicular periodic beam of period $2 p$. Consider the beam of length $p$ between the two perpendicular collisions (see Figure 3).

$(1 \Rightarrow 2$ and 3$)$ Suppose that the midpoint of this beam hits the right-angle vertex. The central symmetry of the rhombus at this collision point implies that the beam itself is centrally symmetric in this point and thus has palindromic code. Furthermore $p$ is even since the symmetry is about an interior point of the rhombus. Label by $I$ the interval of the departure and by $J$ the interval of the arrival. Both intervals are contained in the rhombus labelled 0 , the central symmetry of the beam implies that $I$ and $J$ are centrally symmetric.

$(2 \Rightarrow 1)$ By assumption the initial interval $I$ of the beam and the final interval $J$ fold to the same interval in the triangle Thus the midpoint of $I$ must have period $p$ or have a singular orbit as an orbit in the triangle. It is easy to see that the orbit can not be periodic, since any perpendicular periodic orbit must be twice perpendicular and the collision at time $p / 2$ is not perpendicular. Thus the orbit is singular in the triangle. Since it was nonsingular in the rhombus it hit the right-angled vertex of the triangle.

$(3 \Rightarrow 1)$ Consider the central symmetry of the palindromic beam around its center $c$. This takes the beam to a priori another beam with the same code. Lemma 5 implies that the beams must coincide. Thus the beam is centrally symmetric about its center. Since $p$ is even the point $c$ must lie in the interior of the rhombus visited by the strip at step $p / 2$. However, viewed in the rhombus one sees that the beam can only be centrally symmetric around the center of the rhombus and no other point, thus $c$ must be the center of the rhombus.

We now turn to a detailed analysis of beams which start in a given direction, and either return to that direction or another fixed direction.

Lemma 7. Fix an angle $\theta_{0}$ and consider the angle coding of the billiard with $\theta_{0}$ taken as level 0 . Suppose $N>0$. Consider the set $S_{0}$ of points whose beam of length 2 has code 01 . This set is an interval, partition it into intervals such that for each partition interval there exists $p \geq 1$ such that the initial orbit code $\left\{a_{i}\right\}_{i=0}^{p}$ of all points in the interval does not depend on the points and is such that $a_{0}=0,0<a_{i}<N$ for $i=1, \ldots p-1$ and $a_{p}$ is equal to either 0 or $N$. Then there are at most 
$|N|$ such codes and corresponding subintervals of $S_{0}$. The interiors of these subintervals are pairwise disjoint and their union covers $S_{0}$.

Proof. This lemma has been essentially proven in [GuTr] and somewhat more explicitly in $\mathrm{ScTr}$. I repeat the proof here for completeness. Consider the beam of length two whose initial code is 01. Follow all the orbits in this beam until they reach level $N$ or return to level 0 at some time $p$. For the beam to split, an orbit in the beam must reach a vertex.

This can be seen for example in Figure 3, the beam (not drawn in the picture) just to the left of the beam $A^{+}$has code 01210 (the copy of the rhombus corresponding to last 0 is not drawn in the picture). These two beams are split by a vertex in (the 2nd occurence) of the 1st rhombus.

For each $i$ there is a single vertex $v_{i}$ which can split the beam in rhombus $i$. Since the billiard is invertible this vertex can only be reached from at most one point in the 0th rhombus (without returning to the rhombus). The set $\mathcal{V}:=\mathcal{V}(N):=\left\{v_{i}: 1 \leq i \leq N-1\right\}$ of vertices has cardinality $N-1$, therefore there are at most $N$ distinct beams and thus codes.

Lemma 8. Fix an angle $\theta_{0}$ and consider the angle coding of the billiard with $\theta_{0}$ taken as level 0 . For any $M<0<N$ we can partition level 0 into a finite number of intervals with each interval in one of three classes, $\mathcal{P}:=\mathcal{P}\left(\theta_{0}, M, N\right), \mathcal{M}:=\mathcal{M}\left(\theta_{0}, M, N\right), \mathcal{U}:=\mathcal{U}\left(\theta_{0}, M, n\right)$ such that the orbits of any point in an interval in class $\mathcal{P}$ and $\mathcal{M}$ does not reach level $M$ or $N$. Furthermore

(1) the forward orbit of each boundary point of each interval is singular,

(2) the interior of each of the intervals in $\mathcal{P}$ consists periodic points and the boundaries of each periodic family consists of generalized diagonals,

(3) the intervals in $\mathcal{M}$ consists of one (or several) minimal interval exchange transformations (i.e. on a finite number of intervals!) and the boundary of each minimal component consist of generalized diagonals and

(4) the orbit of each point in the interior of an interval in $\mathcal{U}$ reaches level $M$ or $N$.

We have the following immediate corollary.

Corollary 9. The orbit of every point in level 0 which does not reach level $M$ or $N$ is either singular or returns to level 0.

The classes may well be empty, for example if we choose a direction $\theta_{0}$ for which the surface has no generalized diagonals then both $\mathcal{P}$ and $\mathcal{M}$ 
must be empty. Galperin has shown that for almost all right triangles there exists a $\theta_{0}$ such that the set $\mathcal{M}$ is nonempty Ga.

Proof. Consider the cover of $S_{0}$ defined in the previous lemma and use the subintervals which return to define a partially defined IET on level 0. Do the same for points whose code starts with $0(-1)$. Call the domain of definition $D$. Do the same for the backwards dynamics and remark that these two partially defined maps are inverses of each other where ever they are defined. The total length of the intervals where the forward map is not defined is equal to the total length of the intervals where the backwards map is not define. Thus we can (in an arbitrary manner) complete the definition of the partially defined map to an IET, which we call the ghost map. The ghost IET agrees with the partially defined first return billiard map whenever it was defined.

Since the ghost map is an IET with interval of definition being level 0 . The well known topological decomposition holds, the interval of definition is decomposed into periodic and minimal components, with the boundary of the components consisting of generalized diagonals (see for example [KaHa]).

Any point whose orbit enters an interval on which the ghost dynamics differs from the true dynamics will reach level $M$ or $N$ following the true dynamics. On the other hand the topological decomposition mentioned above can be applied to the point for which the ghost and true dynamics always agree. We remark that a ghost minimal component will, by minimality be either completely contained in $D$ or every orbit will enter an interval on which the ghost dynamics differs from the true dynamics and thus will reach level $M$ or $N$ following the true dynamics.

We next begin the analysis of simple directions. The main reason to analyze such directions is as a "warm-up" for perpendicular directions.

Lemma 10. Suppose $\theta_{0}$ is simple. Then for all integers $M<N$

(1) there exists a nonsingular orbit segment starting in level $M$ and ending in level $N$ and

(2) a nonsingular orbit segment starting in level $N$ and ending in level $M$.

Proof. We first remark that if there is a nonsingular orbit from $M$ to $N$ then since the map is locally an isometry there is a whole interval which maps from $M$ to $N$. Since a.e. point in level $M$ is recurrent this implies that there must be orbits from $N$ to $M$ as well. Thus it suffices to show that either (1) or (2) holds.

Suppose that neither (1) nor (2) holds. Consider the set $S_{M}$ of all points whose code starts with $M(M+1)$ and $S_{N}$ whose code starts with $N(N-1)$. Let $B_{M}^{N}$ be the union of the levels $M+1$ to $N-1$. Since $\theta_{0}$ is simple the previous lemma implies that the backwards orbit 
of every point in $B_{M}^{N}$ must reach $S_{N}, S_{M}$ or be singular. Thus we can partition $B_{M}^{N}$ into intervals whose backwards orbit hit $S_{M}$, intervals whose backwards orbit hits level $S_{N}$, and the backwards singular orbits which divide these intervals.

Since we have assumed there are no orbits connecting levels $M$ and $N$, Corollary 9 implies that if the backwards orbit hits $S_{M}\left(\operatorname{resp} . S_{N}\right)$ then the forward orbit hits level $M$ (resp. level $N$ ). This implies that the points which divide the partition of $B_{M}^{N}$ are also forward singular. Since they are both backwards and forward singular they are part of a generalized diagonal, which contradicts that fact that $\theta_{0}$ is simple. Therefore at least one of (1) or (2) holds, and thus both hold.

Next we prove the following strengthening of Lemma 0 for simple directions. This is the first place that the role of the center of the rhombus becomes apparent.

Proposition 11. Suppose $\theta_{0}$ is simple. Then

(1) there is exactly one code for which $a_{0}=0$ and $a_{p}=N$ and

(2) for all codes for which $a_{0}=a_{p}=0$ the code is a palindrome and $p$ is even.

Proof. Consider the set $S_{0}$ of all points whose code starts with 01 and the set $S_{N}$ of all points whose code starts with $N(N-1)$. Set $S:=S_{0} \cup S_{N}$. Consider the points in $S$ whose orbits arrive at a vertex in $\mathcal{V}$ before returning to level 0 or $N$. The cardinality of $\mathcal{V}$ is $N-1$, thus since $\theta_{0}$ is simple we can apply Lemma 8 to conclude that there are exactly $N-1$ such points. They partition $S$ into $N+1$ intervals. Call the associated beams $A^{(i)}$ where $1 \leq i \leq N+1$. Suppose the $i$ th beam is of length $p(i)$, we denote its code by $\left\{a_{j}^{(i)}\right\}_{j=0}^{p(i)}$.

Consider the set $\mathcal{C}:=\mathcal{C}(N):=\left\{c_{i}: 1 \leq i \leq N-1\right\}$ where $c_{i}$ is the center of the $i$ th rhombus. Consider the orbits which start in $S$ and arrive at one of these centers before returning to level 0 or $N$. By simplicity there are $N-1$ such orbits and they are disjoint from the orbits which start in $S$ and arrive at a vertex in $\mathcal{V}$. The orbit of $c_{i} \in \mathcal{C}$ is symmetric, thus the code of a beam $A^{(i)}$ containing such a point is a palindrome and $p$ is even. The code begins and ends with the same symbol, 0 or $N$, and is strictly positive in between, therefore it can not be a palindrome twice. Thus each of the orbits which arrive at a center are in distinct beams. This implies that each interval contains at most one $c_{i}$ and all but two of the beams contain a center.

We have shown that there are two intervals whose beam does not contain a center. All the other beams start and end on one of the two levels $0, N$ without visiting the other. Thus by Lemma 10, the two beams must be as follows: one connects levels 0 to $N$ and the other levels $N$ to 0 and all other codes are palindromes. 
We would like to extend Proposition 11 to non simple directions, especially to perpendicular directions. There are three places where simplicity was used in the proof, the first is that the pull back of all $v_{i} \in \mathcal{V}$ and all $c_{i} \in \mathcal{C}$ reaches the set $S$. A finer counting argument overcomes this difference. The second is that the pull backs of the $v_{i}$ and $c_{i}$ are disjoint. Finally we applied Lemma 10. We are able to avoid the last two difficulties by assuming that the angle $\alpha$ satisfies $\alpha \in\left(\frac{\pi}{6}, \frac{\pi}{4}\right)$.

Lemma 12. Fix an irrational right triangle $Q$ whose smaller angle $\alpha \in$ $\left(\frac{\pi}{6}, \frac{\pi}{4}\right)$. Consider the angular coding with respect to the perpendicular direction. Then the first return map to level 0 can not be an IET.

Proof. Let $M$ be the invariant surface containing the perpendicular direction. Consider the set $G$ of generalized diagonals which are contained in the invariant surface $M$. Note that since $Q$ is irrational the set $G$ can be infinite. Each generalized diagonal is a segment in $M$. Several generalized diagonals can intersect at a singular point, thus the set $G$ has the structure of a graph, not necessarily connected.

Suppose the conclusion is not true. Then, the set of perpendicular periodic orbits form a finite union of annuli $A \subset M$. We have $A \neq M$ since the area of $M$ is infinite and the area of $A$ is finite. Thus $A$ must have a boundary which must consist of a finite union of generalized diagonals. We will analyze $G$ to show that this boundary must be empty.

The boundary of $A$ consists of periodic loops. A periodic loop is a finite union of generalized diagonals which is periodic. We can think of a periodic loop as a (one-sided) boundary of a family of periodic orbits. The idea of the proof is to analyze all possible periodic loops which could possibly form the boundary of $A$, i.e. those that have perpendicular periodic orbits on at least one side. Our analysis will show that in each case they must have perpendicular periodic orbits on both sides and thus they can not form part of the boundary of $A$.

A periodic loop $g$ is called simple if it consists of a single generalized diagonal, or equivalently the connected component of $G$ containing $g$ is $\{g\}$. Let $L$ be the horizontal diagonal of the rhombus.

Consider the orbit $g_{L}$ starting from the left endpoint of $L$. Since $\pi / 2<3 \alpha<3 \pi / 4$ the symbolic coding of $g_{L}$ is 010 , and $g_{L} g_{L}$ hits $L$ again in an interior point without hitting any other vertex before (see Figure 5). Thus $g_{L}$ is a simple periodic loop. Since $g_{L}$ hits $L$ at an interior point it has perpendicular periodic orbits on both sides of it and can not form part of the boundary of $A$. Similarly the perpendicular diagonal starting at the right end point of $L$ can not be part of the boundary of $A$.

Consider any connected component $G^{\prime}$ of $G$ such that $G^{\prime} \cap \bar{A} \neq \emptyset$. This implies that there is at least one generalized diagonal $g_{1} \in G^{\prime}$ 


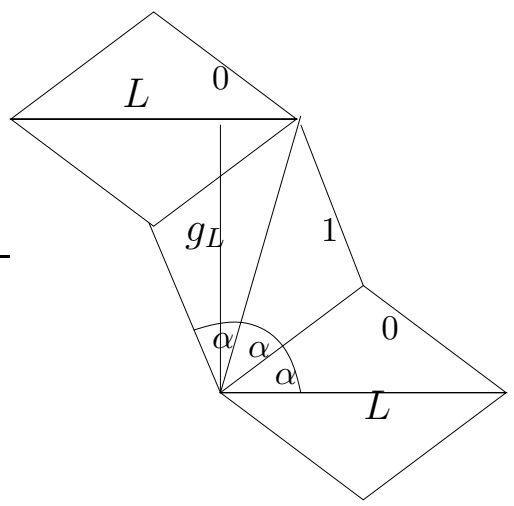

Figure 5. $g_{L}$ is a simple periodic loop.

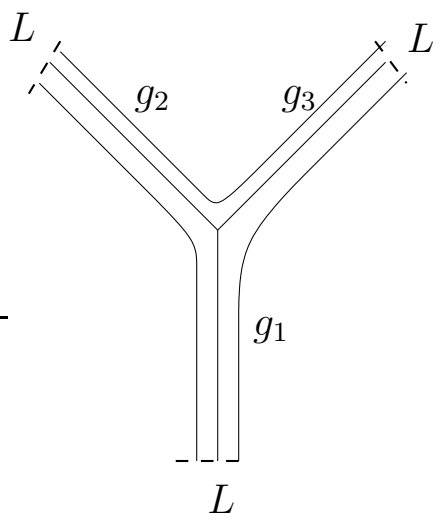

FiguRE 6. $G^{\prime}$ consisting of three periodic loops, $\left\{g_{1}, g_{2}\right\}$, $\left\{g_{2}, g_{3}\right\}$, and $\left\{g_{3}, g_{1}\right\}$.

whose orbit hits $L$ perpendicularly, and thus we have a perpendicular periodic loop $g^{\prime}=g_{1}, \ldots, g_{n}$ in $G^{\prime}$. Since we have finished treating it, we can assume $g_{L} \notin G^{\prime}$ and thus $g_{1}$ hits $L$ in an interior point.

I claim that the periodic loop hits $L$ again perpendicularly at another interior point of $L$. First of all $g^{\prime}$ can not hit $L$ again at an endpoint by assumption. Start flowing $g^{\prime}\left(g_{1}\right)$ forwards and backwards from $L$, this is a symmetric orbit in $M$. At exactly half of $g^{\prime}$ 's (minimal) period, the forward and backwards orbits meet. The meeting point must also be a symmetry point of the orbit. The only points of symmetry available are perpendicular reflections (in our case since $Q$ is irrational the only axis of reflection in $M$ is $L$ ) and central symmetries through copies of the center of $Q$. I claim that the only possibility for the second symmetry of $g$ is that it must hit $L$ a second time at an different interior point of $L$. First it can not hit a central symmetry point since such points are isolated, while the second symmetry holds for the whole periodic family which $g^{\prime}$ bounds. Furthermore $g^{\prime}$ can not hit $L$ a second time at the same end point, in fact assuming this holds halves the period of $g^{\prime}$, a contradiction of the definition of minimal period. 
Thus both sides of $g_{1}$ must consist of periodic perpendicular orbits (see Figure 6). If $g^{\prime}$ is simple then both sides of $g^{\prime}$ consist of periodic perpendicular orbits and $g^{\prime}$ can not be part of the boundary of $A$. If $g^{\prime}$ is not simple then the "other" side of $g^{\prime}$ is also a periodic loop, for which, by the same reasoning, bounds perpendicular period orbits. Since $A$ is a finite union of annuli, there can only be a finite number of perpendicular periodic loops. Thus, by exhaustion, all of $G^{\prime}$ consists of perpendicular periodic loops, and can not form part of the boundary of $A$. This contradiction completes the proof.

Next we prove the analog of Proposition 11 for perpendicular directions.

Proposition 13. Fix an irrational right triangle $Q$ whose smaller angle $\alpha \in\left(\frac{\pi}{6}, \frac{\pi}{4}\right)$. Consider the angular coding with respect to the perpendicular direction, then

(1) there is exactly one code for which $a_{0}=0$ and $a_{p}=N$ and

(2) for all codes for which $a_{0}=a_{p}=0$ the code is a palindrome and $p$ is even.

Proof. We consider the same notation as in the proof of Proposition 11, the difference being that generalized diagonals exist. Suppose for the moment that no generalized diagonal passes through both $\mathcal{C}$ and $\mathcal{V}$. Note that by symmetry a perpendicular orbit can reach only a single $c \in \mathcal{C}$. Let $J \leq N-1$ be the cardinality of the points in $S$ whose orbits arrive at a vertex in $\mathcal{V}$ before returning to level 0 or $N$. They partition the $S$ into $J+2$ intervals and corresponding beams $A^{(i)}$. Let $K$ be the cardinality of the set of orbits which start in $S$ and arrive $\mathcal{C}$. Consider the beam $A^{(i)}$ containing such an orbit, arguing as in the simple case that each center is in a different beam yields $K \leq J+2$.

If no orbit from $S$ reaches $c_{i}$ before returning level 0 or $N$, then the orbit of $c_{i}$ never reaches rhombus 0 or $N$. Thus by Lemma 8 it's orbit is periodic or its orbit closure is a minimal IET. In either case since the map is a local isometry we can associate $c_{i}$ with a vertex $v_{j(i)} \in \mathcal{V}$ such that no orbit from $S$ reaches this vertex. Furthermore one generalized diagonal can not be the complete boundary to two minimal or periodic components of an IET. Thus for a set of cardinality $L$ of such $c_{i}$ we must have at least $L$ distinct such vertices $v_{j(i)}$. This implies $N-1-J \geq N-1-K$, or $J \leq K$. We have shown that $J \leq K \leq J+2$, i.e. the number of beams which do not contain a center is at most 2 . These two beams are the only candidates for beams connecting level 0 to $N$ and $N$ to 0 .

Now we deal with the case when there is an orbit passing through both $\mathcal{C}$ and $\mathcal{V}$. Note that any orbit which starts perpendicularly and arrives at $C$ before $\mathcal{V}$, returns, by the central symmetry to a perpendicular collision and is thus simple. Thus we have reduced to the case 
when an orbit visits $\mathcal{V}$ before $\mathcal{C}$. By the central symmetry it is a generalized diagonal. The counting argument above works with the following differences. First of all we consider this generalized diagonal as a degenerate beam $A^{(i)}$. For this degenerate beam we interpret $J$ and $K$ as follows. Since $A^{(i)}$ visits a single $c \in \mathcal{C}$ it contributes one to $K$ and since $J+2$ is the number of beams it contributes one to $J$. With these modifications the inequality $J \leq K \leq J+2$ remains true and as above we conclude that all but at most two of the beams contain a center. By the central symmetry around the center the code of each such beam must satisfy $a_{0}=a_{p} \in\{0, N\}$.

Next we need to show that exactly two exceptional beams exist. If no exceptional beam exists then Lemma 12 yields an immediate contradiction. If one exceptional beam exists, if $a_{0}=a_{p}$ then again Lemma 12 yields an immediate contradiction. In the other cases $\left(a_{0}=\right.$ $0, a_{p}=N$ or $a_{0}=N, a_{p}=0$ ) the points of the exceptional beam can not be recurrent, which is a contradiction of the fact that almost every point is recurrent. Thus exactly two exceptional beams exist. All the other beams contain a center and thus are palindromes with $p$ even.

Finally we need to show that in fact one of the two beams satisfies $a_{0}^{1}=0, a_{p}^{1}=N$ and the other satisfies $a_{0}^{2}=N, a_{p}^{2}=0$. If we think of the beams as marked (1st and 2nd) then there are 16 possible cases depending on of $a_{i}^{j}=0$ or $N$ for $j=1,2$ and $i=0, p$. Two of these cases are good, we must eliminate all the 14 other cases.

Since almost every point is recurrent we can not have a beam starting with 0 and ending with $N$ without the other beam connecting level $N$ to level 0 , this eliminates 6 cases.

To be able to apply Lemma 12 repeat the construction for points which are perpendicular to the leg of the triangle but whose code is strictly negative before returning to level 0 or $-N$ to produce two addition exceptional beams with codes $b_{i}^{j}$, which are centrally symmetric to the two original ones in the sense that $a_{i}^{j}=-b_{i}^{j}$ for $j=1,2$. A direct application of Lemma 12 eliminates the the remaining 8 bad cases when there is no beam starting in 0 ending in $N$.

Proof of Theorem 1. The theorem follows immediately for by combining Lemma 6 and Propostion 13.

Proof of Theorem 2. We start with the simple case. First consider orbits whose code starts with 01 . Any such nonsingular nonrecurrent orbit must be contained in the intersection $\cap_{N \geq 1} B^{i_{0}(N)}$ where $B^{i_{0}(N)}$ is the unique interval which satisfy alternative (1) for fixed $N \geq 1$ of Proposition 11. Consider the initial segment of these beams, these intervals are clearly nested, thus there is at most one interval or a single point in the intersection of their closures. Since the set of nonrecurrent orbits has measure zero it consists of at most a single point. The orbits 
of this point is either a positive escape orbits or singular. Similarly we find a single positive escape orbit starting on any level $N$. This escape orbit must, by uniqueness, coincide with the one starting on level 0 .

A symmetric argument holds for the backwards escape orbit, by starting with points whose code commences with $0-1$.

We turn to the perpendicular case. The fist difference to the simple case is that we replace Proposition 11 by Proposition 13. We immediately obtain that there is at most one positive escape orbit starting on level 0 . To show that this is the unique positive escape orbit on the whole surface we argue like in the proof of Propostion 13. Fix $M>N>0$. The first part proof goes through for levels $M$ and $N$, i.e. we know that there are at most two exceptional beams. We need to conclude that they exist and that they connect levels $M$ and $N$ to each other without applying Lemma 12. This follows immediately from the fact that there is a beam connecting level 0 to $N$ and a beam connecting level $N$ to level 0 .

By symmetry, the positive and negative escape orbits must be the forward and backwards part of the same orbit.

Proof of Theorem 3. The proof is similar to the proof of Lemma 12. The set of perpendicular periodic orbits form a countable union of annuli $A \subset M$. If $A \neq M$ then $A$ must have a boundary which must consist of generalized diagonals and escape orbits. Besides the escape orbit, there is an additional difficulty that the relevant part of the graph of generalized diagonals on $M$ need not be finite.

The argument that a finite connected component $G^{\prime}$ satisfying $G^{\prime} \cap$ $\bar{A} \neq \emptyset$ can not form part of the boundary of $A$ is the same as in the proof of Lemma 12. This completes the proof in the rational case.

Next suppose that the escape orbit exists, i.e. is nonsingular. Denote this orbit by $e$. By construction, the orbit $e$ starts on level 0 at a point $e_{0}$ which is an interior point of $L$. Thus we can find a sequence of perpendicular periodic orbits which approaches $e$ from the left and a sequence which approaches $e$ from the right, and $e$ can not form part of the boundary of $A$.

The remaining case is $G^{\prime}$ infinite. There are two subcases, either every edge in the graph has periodic perpendicular orbits on both sides or not. In the first case we argue as in the proof of Lemma 12, using induction instead of exhaustion to complete the proof. In the second case the (unique) escape orbit $e$ is infact singular and contained in $G^{\prime}$, see Figure 7 . Thus the one side of $e$ bounds perpendicular periodic families, while the other side is a limit or perpendicular periodic families.

The method of proof also allows to verify the Boshernitzan conjecture for an infinite collection of rational right triangles, those where 


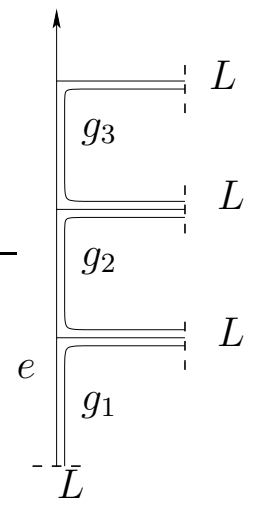

FiguRE 7. An infinite connected component $G^{\prime}$ which includes the escape orbit.

the perpendicular orbits starting at the (non-right angle) verteces are simple periodic loops.

Proof of Theorem 1 . For irrational triangles this follows immediately from Theorem 3 since the surface $M$ is dense in the full phase space. For rational triangles the result was established in [BoGaKrTr].

\section{EXTENSIONS}

I would like to remark on the assumption $\frac{\pi}{6}<\alpha<\frac{\pi}{4}$. This is a technical assumption which guarantees that the orbits starting at the endpoints of $L$ are simple periodic loops. This is the key condition which is used in the proof of the theorems. It seems likely this condition holds for an open set of full measure of parameters $\alpha$, but to prove this seems messy and complicated.

\section{AcKnowledgements}

I profitted greatly from discussions with Pascal Hubert.

\section{REFERENCES}

[Bo] M. Boshernitzan, Billiards and rational periodic directions in polygons, Amer. Math. Monthly 99 (1992) 522-529.

[BoGaKrTr] M. Boshernitzan, G. Galperin, T. Krüger, and S. Troubetzkoy Periodic billiard orbits are dense in rational polygons, Transactions AMS 350 (1998) 3523-3535.

[CiHaKo] B. Cipra, R. Hanson and A. Kolan, Periodic trajectories in right triangle billiards, Phys. Rev. E 52 (1995) 2066-2071.

[Ga] G. Galperin, Non-periodic and not everywhere dense billiard trajectories in convex polygons and polyhedrons, Comm. Math. Phys. 91 (1983), 187-211.

[GaStVo] G. Galperin, A. Stepin and Ya. Vorobets, Periodic billiard trajectories in polygons: generating mechanisms, Russian Math. Surveys 47 (1992) 5-80. 
[GaZv] G. Galperin and D. Zvonkine, Periodic billiard trajectories in right triangles and right-angled tetrahedra, Regular and Chaotic Dynamics 8 (2003) 29-44.

[Gu1] E. Gutkin, Billiard in polygons, Physica D, 19 (1986) 311-333.

[Gu2] E. Gutkin, Billiard in polygons: survey of recent results, J. Stat. Phys., 83 (1996) 7-26.

[GuTr] E. Gutkin and S. Troubetzkoy, Directional flows and strong recurrence for polygonal billiards, in Proceedings of the International Congress of Dynamical Systems, Montevideo, Uruguay, F. Ledrappier et. al. eds. (1996) 21-45.

[KaHa] A. Katok and B. Hasselblatt, Encyclopedia of Mathematics and its Applications, 54. Cambridge University Press, Cambridge, 1995.

[Ma] H. Masur, Closed trajectories of a quadratic differential with an application to billiards, Duke Math. Jour. 53 (1986) 307-313.

[MaTa] H. Masur and S. Tabachnikov, Rational billiards and flat structures, Handbook of dynamical systems, Vol. 1A, 1015-1089, North-Holland, Amsterdam, 2002.

[Ru] T. Ruijgrok, Periodic orbits in triangular billiards Acta Physica Polonica B 22 (1991) 955-981.

[ScTr] J. Schmeling and S. Troubetzkoy Inhomogeneous Diophantine approximation and angular recurrence for polygonal billiards Math. Sb. 194 (2003) 295-309.

[Ta] S. Tabachnikov, Billiards, "Panoramas et Syntheses", Soc. Math. France (1995).

[Tr] S. Troubetzkoy, Recurrence and periodic billiard orbits in polygons, preprint.

Centre de physique théorique, Federation de Recherches des Unites de Mathematique de Marseille, Institut De mathématiques De Luminy and, Université de la Méditerranée, Luminy, Case 907, F-13288 MarSEILle Cedex 9, France

E-mail address: troubetz@iml.univ-mrs.fr

$U R L:$ http://iml.univ-mrs.fr/ troubetz/ 